\title{
Simulação in situ: ganho da autoconfiança de profissionais de enfermagem na parada cardiopulmonar
}

\author{
In situ simulation: the gain of self-confidence by nursing professionals during \\ cardiopulmonary arrests
}

Mariana Nunes Almeida ${ }^{1}$, Tayse Tâmara da Paixão Duarte ${ }^{1}$, Marcia Cristina da Silva Magro ${ }^{1}$

\begin{abstract}
Objetivo: comparar a simulação in situ em relação à aula didática tradicional, no ganho da autoconfiança de profissionais da equipe de enfermagem, em situação de parada cardiopulmonar. Métodos: estudo quasiexperimental, não equivalente, desenvolvido em unidade de terapia intensiva e pronto socorro de hospital geral. A intervenção foi a simulação in situ. Para coleta de dados, adotou-se questionário de caracterização profissional, a autoconfiança avaliada pela escala Self-Confidence Scale. Considerou-se significativo resultados com $\mathrm{p} \leq 0,050$. Resultados: participaram 37 profissionais de enfermagem. 0 grupo experimental pré-simulação se mostrou menos autoconfiante $(3,4 \pm 0,6)$ quando comparado ao grupo controle $(3,9 \pm 0,7)$. Entretanto, 30 dias após a intervenção, o grupo experimental mostrou a manutenção de ganho significativo da confiança $(\mathrm{p}=0,040)$. Conclusão: a autoconfiança dos profissionais de enfermagem, direcionada à assistência ao paciente em parada cardiopulmonar, melhorou significativamente com emprego da simulação in situ, quando comparada à aula didática tradicional, dialogada.
\end{abstract}

Descritores: Simulação; Treinamento por Simulação; Confiança; Cuidados Críticos; Equipe de Enfermagem.

Objective: to compare in situ simulation to the traditional didactic methodology, regarding the gain of selfconfidence of nursing team professionals regarding cardiopulmonary arrest. Methods: quasi-experimental nonequivalent study, developed in an intensive care unit and emergency room of a general hospital. The intervention was an in situ simulation. For data collection, a professional characterization questionnaire was used, and the self-confidence aspect was assessed by the Self-Confidence Scale. Results with $p \leq 0.050$ were considered significant. Results: 37 nursing professionals participated. The pre-simulation experimental group was less self-confident ( $3.4 \pm 0.6)$ when compared to the control group (3.9 \pm 0.7$)$. However, 30 days after the intervention, the experimental group showed a significant gain in confidence $(\mathrm{p}=0.040)$. Conclusion: the self-confidence of nursing professionals with regards to assisting patients in cardiopulmonary arrest improved significantly with the use of an in situ simulation, when compared to the traditional didactic methodology, through dialogue.

Descriptors: Simulation; Simulation Training; Trust; Critical Care; Nursing, Team.

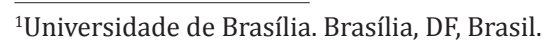




\section{Introdução}

A autoconfiança é uma habilidade não técnica, capaz de influenciar as ações desempenhadas pela equipe de enfermagem, principalmente em situações complexas, ganhando relevância no cenário de urgên$\operatorname{cia}^{(1)}$.

Os sucessos, insucessos e as dificuldades são condições que exigem do profissional capacidade de adaptação e enfrentamento, para que haja superação e alcance de desfecho positivo ${ }^{(2)}$. Nesse cenário, a autoconfiança, quando em consonância com a resiliência, possibilita melhor adaptação para enfrentamento dos desafios ${ }^{(3)}$.

Programas de treinamento baseados em simulação podem se utilizar de experiências clínicas direcionadas, para padronizar intervenções e preencher lacunas identificadas na autoconfiança e atuação prática de profissionais em situações críticas ${ }^{(4)}$.

A simulação clínica, no entanto, configura-se como estratégia disparadora do contato, com cenas reais, por meio de experiências guiadas ${ }^{(5)}$. Os programas interativos de simulação são ferramentas capazes de disponibilizar recursos realistas reproduzidos por manequins, anatomicamente semelhantes à pessoa humana que, ao expressar respostas fisiológicas frente a intervenções reais, permitem a interação, mediada pela comunicação verbal ou não verbal, tanto do instrutor como do participante ${ }^{(6)}$.

A simulação enquanto ferramenta de capacitação contribui para o desenvolvimento do raciocínio clínico, sendo, portanto, aplicável durante o curso de graduação e, também, relevante para melhoria do desempenho profissional durante o programa de educação permanente ${ }^{(5)}$. Nos dias atuais, dada a necessidade do desenvolvimento da autoconfiança do profissional, a simulação funciona como excelente recurso ao alcance do cuidado seguro e individualizado(7).

A simulação, quando disponibilizada no próprio ambiente de trabalho, por meio de recursos e equipamentos que proporcionam à equipe de saúde experiência próxima à realidade, denomina-se de si- mulação in situ ${ }^{(8)}$. Nesta perspectiva, afigura-se como veículo ao desenvolvimento e aperfeiçoamento da autoconfiança, contribuindo para aprimoramento da equipe, especialmente no âmbito do cuidado crítico e de $\operatorname{risco}^{(9)}$.

Cada vez mais, a capacitação profissional se torna imprescindível nos setores de assistência à saúde. Métodos inovadores de treinamento que melhorem o conhecimento e a confiança dos provedores podem compensar as deficiências na experiência clínica $^{(10)}$. Há reconhecimento do papel da simulação in situ no treinamento e na avaliação de habilidades técnicas e não técnicas, com reforço da autoconfiança, comunicação e trabalho em equipe ${ }^{(11)}$, ao considerar que facilita a observação de como o cuidado acontece em tempo real, em vez de apenas especular o que pode acontecer ou como deveria acontecer a partir de ferramentas didáticas.

A simulação no ambiente de trabalho real foi identificada como particularmente valiosa, porque reúne os elementos da equipe de atendimento e do ambiente ${ }^{(12)}$. Pesquisadores reconheceram que o papel da simulação in situ vai além do treinamento e da avaliação de habilidades técnicas e não técnicas, sobretudo, enfatizaram as maneiras exclusivas pelas quais essa estratégia poderia ser usada para avaliar a competência do sistema e identificar condições latentes que predispõem ao erro médico ${ }^{(11)}$.

Frente ao exposto, objetivou-se comparar a simulação in situ em relação à aula didática tradicional, no ganho da autoconfiança de profissionais da equipe de enfermagem, em situação de parada cardiopulmonar.

\section{Métodos}

Trata-se de estudo quasi-experimental, não equivalente, com pré e pós-testes. 0 estudo foi desenvolvido em unidade de terapia intensiva geral para adulto e salas amarela e vermelha de pronto socorro de hospital geral público do Distrito Federal, Brasil.

A população do estudo foi constituída de 37 
profissionais de enfermagem da unidade de terapia intensiva e de 43 do pronto socorro, a época da coleta de dados. A amostra foi não probabilística, de conveniência e constituiu-se de cinco enfermeiros e 13 técnicos de enfermagem da unidade de terapia intensiva (grupo experimental) e cinco enfermeiros e 14 técnicos de enfermagem do pronto socorro (grupo controle). Quando houve necessidade de comparação entre grupos, os instrumentos com ausência de alguma resposta foram excluídos, isto aconteceu em duas situações, o que resultou no tamanho amostral final de 37 profissionais. As demais perdas amostrais decorreram de férias ou licença trabalhista ou, ainda, recusa em participar do estudo.

A intervenção foi a implementação da estratégia de simulação in situ e o controle foi a aula didática dialogada (tradicional), ambas direcionadas à assistência de enfermagem ao paciente em parada cardiopulmonar.

Foram incluídos profissionais de enfermagem com pelo menos seis meses de experiência profissional e excluídos aqueles que não exerciam assistência direta ao paciente ou atuavam em ambos os setores; enfermeiros substitutos e/ou afastados, residentes e estagiários de enfermagem.

A coleta de dados ocorreu de dezembro de 2017 a março de 2018, por meio de visitas realizadas aos profissionais no ambiente de atuação, conforme distribuição da escala de trabalho mensal.

Na fase pré-simulação in situ, realizou-se validação do cenário "assistência ao paciente em parada cardiopulmonar" por três juízes (docentes com expertise na área, conforme dados do currículo Lattes) que analisaram e avaliaram a coerência, aparência, realismo, disponibilidade de recursos e condução de debriefing ${ }^{(13)}$ (discussão da cena vivenciada entre pesquisador e participantes), em laboratório de simulação de instituição de ensino superior pública. Os ajustes sugeridos foram atendidos e, no segundo momento ,repetiu-se a validação no setor do hospital onde foi desenvolvido o estudo, para ajustes da proposta de simulação ao próprio ambiente.
Sequencialmente, aplicou-se tanto o questionário de identificação, constituído dos dados demográficos e profissionais, como a versão portuguesa da Self-Confidence Scale ${ }^{(14)}$, desenvolvida por Frank Hicks e versão publicada, posteriomente ${ }^{(15)}$. 0 desenvolvimento desta teve como objetivo central avaliar a variável autoconfiança, subdividida em quatro dimensões. A escala consiste na lista de doze itens, com respostas tipo Likert, com cinco possibilidades: nada confiante, pouco confiante, confiante, muito confiante e extremamente confiante. Os diferentes itens identificam a capacidade do estudante formando para: (1) reconhecer sinais e sintomas de alterações nas referidas áreas, (2) avaliar com precisão o doente, (3) intervir apropriadamente e (4) avaliar a eficácia das intervenções implementadas, nas áreas respiratória, cardíaca e neurológica. 0 alpha de Cronbach obtido na versão portuguesa (vp) foi de $0,918^{(14)}$. Destaca-se que os 12 itens da escala foram adaptados para profissionais de enfermagem ativos em cenários de assistência ao paciente crítico.

Aos profissionais da unidade de terapia intensiva (grupo experimental), implementou-se a simulação in situ (grupo experimental), por meio de simulador de paciente de média fidelidade da Laerdal ${ }^{\circledR}$, capaz de reproduzir as condições de um paciente em parada cardiopulmonar. A reprodução realística foi possível, por meio da participação de dois assistentes de pesquisa (estudantes/monitores capacitados) que auxiliaram a projeção dos parâmetros hemodinâmicos vitais e reproduziram a voz do simulador.

Ao término, realizou-se o debriefing, sessão de discussão e reflexão entre os profissionais e o facilitador da cena vivenciada. As simulações foram filmadas e ocorreram em quarto da própria unidade de terapia intensiva, de forma a surpreender os profissionais de enfermagem e garantir sigilo das sessões do cenário. Assim, o fato de os cenários das sessões de simulação não serem anunciados possibilitou a redução do efeito de adesão, ou seja, a repetição da mesma resposta pelos profissionais, quando expostos à mesma situação ${ }^{(16)}$. 
Para fins de comparação, os profissionais de enfermagem do grupo controle foram dispensados no início do plantão, conforme cronograma de liberação previamente acordado com a chefia imediata de enfermagem, para participação em exposição didática (aula dialogada) de abordagem pedagógica tradicional referente ao tema parada cardiopulmonar. A pesquisadora implementou a proposta em espaço específico, mas no próprio pronto socorro, a fim de minimizar interrupções prolongadas do processo assistencial, mediada por multimídia e material educativo e durante 30 minutos abordou sobre conceito e protocolo de atendimento da parada cardiopulmonar, conforme diretrizes da Associação Americana de Car$\operatorname{diologia}^{(17)}$.

Dessa forma, o resultado da intervenção (grupo experimental), quando comparado ao grupo controle (estratégia pedagógica de ensino tradicional), possibilitaria verificar se a simulação in situ contribuiria para maior ganho da autoconfiança pelos profissionais de enfermagem, frente a situações de parada cardiopulmonar, quando comparada à estratégia pedagógica tradicional, conforme descrito no objetivo deste estudo.

Tanto para o grupo experimental como para o controle, reaplicou-se a escala de autoconfiança (Self-Confidence Scale) um mês (30 dias) após as intervenções, para avaliação do nível de autoconfiança dos profissionais em assistir a parada cardiopulmonar, mesmo após um período prolongado da implementação de ambas estratégias (simulação in situ e aula dialogada).

Realizou-se análise descritiva, por meio de medidas resumo (média e mediana) e de dispersão (desvio padrão e percentis 25 e 75). Para testar a distribuição da amostra, aplicou-se o teste Kolmogorov Smirnov, mas não se confirmou a hipótese de normalidade, assim, aplicaram-se os testes não paramétricos de Mann-Whitney e Wilcoxon Ranks, conforme apropriado.

Para análise dos dados, adotou-se o programa estatístico Statistical Package for Social Sciences, ver- são 23, e considerou-se significativo os resultados com $\mathrm{p} \leq 0,050$.

A pesquisa foi aprovada pelo Comitê de Ética em Pesquisa da Faculdade de Ciências da Saúde da Universidade de Brasília e da Fundação de Ensino e Pesquisa em Ciências da Saúde, conforme parecer: 2.357.399/17 e Certificado de Apresentação para Apreciação Ética no 47071915.0.0000.0030. Os participantes assinaram o Termo de Consentimento Livre e Esclarecido e o Termo de Autorização de Imagem e Som.

\section{Resultados}

Dos 37 profissionais de enfermagem distribuídos entre os grupos controle e experimental, a maioria $(n=26)$ era do sexo feminino (70,3\%), com idade mediana de $37(32$ - 40) anos. Entre estes, quase a metade tinha concluído o curso de graduação, 15 (40,5\%), e ainda 10 (27,0\%) possuíam nível médio, 11 (29,7\%) possuíam especialização (pós-graduação lato sensu) e somente um $(2,7 \%)$ o título de mestre (pós-graduação stricto sensu). 0 tempo de atuação dos profissionais da unidade de terapia intensiva foi pouco superior aos do pronto socorro (4 vs. 3 anos). Do total de profissionais, $18(48,6 \%)$ declararam que quando admitidos, participaram de treinamento para o exercício de funções. Aponta-se que a maioria, 19 (51,4\%), informou experiência com a estratégia de simulação como modelo de ensino, durante processo de capacitação profissional.

Ao considerar que a Self-Confidence Scale varia de 1 a 5 pontos, sendo 1 nada confiante e 5 extremamente confiante, os profissionais do grupo experimental, inicialmente (pré-intervenção), mostraram-se menos autoconfiante, 3,4 (3,0 - 3,8), quando comparados ao grupo controle, 4,1 $(3,2-4,3)(\mathrm{p}=0,040)$. Entretanto, 30 dias após a intervenção, o grupo experimental mostrou a manutenção de ganho significativo da confiança de $3,4(3,0-3,8)$ para $3,7(3,2-4,1)$, $\mathrm{p}=0,040$, condição não identificada no grupo controle ao constatar que a autoconfiança dos profissionais desse grupo praticamente não se modificou da fase 
inicial para final $[3,9 \pm 0,7$ vs $4,0 \pm 0,9]$.

A autoconfiança no grupo experimental para assistência ao paciente em parada cardiopulmonar foi significativamente maior no grupo de profissionais de enfermagem mais jovens (idade $<35$ anos) em relação àqueles com idades $\geq 35$ anos, 30 dias após intervenção (retenção de conhecimento) ( $\mathrm{p}=0,040$ vs $\mathrm{p}=0,400$ ), mas no grupo controle, não se identificou diferença significativa (Tabela 1).

Tabela 1 - Comparação da autoconfiança entre as diferentes faixas etárias dos profissionais de enfermagem para cada fase do estudo dos grupos experimental e controle

\begin{tabular}{lcccc}
\hline & \multicolumn{4}{c}{ Fases } \\
\cline { 2 - 5 } $\begin{array}{l}\text { Idade } \\
\text { (anos) }\end{array}$ & $\begin{array}{c}\text { Grupo Experimental Mediana } \\
\mathbf{( 2 5 - 7 5 )}\end{array}$ & $\begin{array}{c}\text { Grupo Controle Mediana } \\
\mathbf{( 2 5 - 7 5 )}\end{array}$ \\
\cline { 2 - 5 } & $\begin{array}{c}\text { Pré-intervenção } \\
\text { Retenção }\end{array}$ & Pré-intervenção & Retenção \\
\hline 35 & $3,6(3,5-3,8)$ & $3,9(3,8-4,3)$ & $4,1(3,5-4,3)$ & $4,3(3,0-4,8)$ \\
$\geq 35$ & $3,2(3,0-3,5)$ & $3,5(3,1-3,8)$ & $4,0(3,3-4,7)$ & $4,2(3,0-4,7)$ \\
p* & 0,400 & 0,040 & 0,700 & 0,800 \\
\hline *Teste Mann-Whitney & & &
\end{tabular}

\section{Discussão}

Os resultados deste estudo confirmaram que a estratégia de simulação in situ proporcionou aos profissionais de enfermagem maior ganho da autoconfiança para atendimento de uma parada cardiopulmonar, quando comparada à capacitação por meio do ensino tradicional, aqui reconhecida como aula dialogada.

A simulação in situ representa estratégia relativamente inovadora que proporciona a vivência de experiências clínicas reais no próprio ambiente de trabalho, de forma controlada, a fim de minimizar desafios futuros, renovar o conhecimento previamente adquirido, aumentar a autoconfiança do profissional, aprimorar habilidades e, principalmente, identificar fatores de risco para segurança do paciente ${ }^{(8-9)}$. Melhorar a segurança do paciente tem se mostrado imprescindível para todas as organizações e equipes de saúde, principalmente aquelas que trabalham com cuidados aos pacientes críticos $^{(18)}$.

Um diagnóstico preciso e imediato e o gerenciamento de pacientes em situações de emergência ou gravemente doentes são de extrema importância. Embora muitos protocolos sejam assumidos para auxiliar esse processo, tem sido limitada a ênfase em habilidades não clínicas de cuidados de saúde, como a autoconfiança por profissionais, como enfermeiros e técnicos de enfermagem, durante emergências. Entre essas habilidades, a autoconfiança, como identificada no presente estudo, permanece como fator imprescindível nas tomadas de decisão e ocupa papel crucial no desempenho profissional ${ }^{(19)}$.

Dada a complexidade dos pacientes em parada cardiopulmonar, torna-se imperativo que enfermeiros e técnicos de enfermagem tenham confiança em conhecimentos e habilidades para verificar quando a situação estiver se agravando e, então, agir adequadamente, a fim de prevenir e tratar doenças, reduzindo a readmissão hospitalar. Portanto, percebe-se que profissionais de enfermagem, quando confiantes, melhoram o desempenho, mesmo em situações de alta complexidade ${ }^{(19)}$.

Estudo desenvolvido em universidade da Austrália mostrou que a confiança no processo de cuidar foi inversamente associada à idade, indicando que os maiores níveis de confiança foram identificados em estudantes de enfermagem mais jovens ${ }^{(20)}$. No presente estudo, embora tenha se desenvolvido com profissionais da equipe de enfermagem, essa relação se manteve.

0 treinamento em equipe foi proclamado como método capaz de melhorar a segurança e a qualidade da assistência à saúde. Em unidade de terapia intensiva, ambiente de alta tecnologia, enfermeiros atuam em equipes disciplinares e interdisciplinares, frente às condições de rápida mudança, como a parada cardiopulmonar. Falhas no desempenho da equipe em relação às habilidades não técnicas, como a autoconfiança, são fatores que contribuem frequentemente para ocorrência de incidentes ${ }^{(13)}$. 
Nessa perspectiva, reforça-se que a simulação representa ferramenta de ensino e aprendizagem adotada em situações de treinamento e capacitação de emergência, capaz de proporcionar aumento da autoconfiança e do desenvolvimento de raciocínio clínico $^{(5)}$.

A pesquisa mostrou como limitações a indisponibilidade de alguns profissionais em participar do estudo, embora a simulação in situ tenha sido disponibilizada em diferentes horários. Apesar disso, o uso da simulação in situ revelou-se como estratégia potencial e significativa ao ganho da autoconfiança dos profissionais de enfermagem, considerando a repercussão desta sobre o desenvolvimento de habilidade não técnica, como a autoconfiança, no cuidado direto e segurança ao paciente, enquanto diferencial para o sucesso do cuidado.

\section{Conclusão}

A autoconfiança dos profissionais de enfermagem, direcionada à assistência ao paciente em parada cardiopulmonar, melhorou expressivamente com o emprego da simulação in situ, quando comparado à aula didática tradicional, dialogada.

Os achados deste estudo sugerem que o investimento em educação baseada em simulação tende, de forma evidente, melhorar, de um lado, os planos de cuidados, a qualidade e a segurança do paciente, do outro, a autoconfiança dos profissionais de enfermagem, ambos achados em situações de emergência.

\section{Agradecimentos}

À Fundação de Apoio à Pesquisa do Distrito Federal, por concessão de financiamento para o desenvolvimento do projeto de pesquisa.

\section{Colaborações}

Almeida MN e Magro MCS contribuíram com concepção e projeto, análise e interpretação dos da- dos. Duarte TTP colaborou com redação do artigo, revisão crítica relevante do conteúdo intelectual e aprovação final da versão a ser publicada.

\section{Referências}

1. Mazzo A, Martins JCA, Jorge BM, Batista RCN, Almeida RGS, Henriques FMD, et al. Validation of the self-confidence scale of nursing care in urinary retention. Rev Latino-am Enfermagem. 2015; 23(5):814-20. doi: http://dx.doi. org/10.1590/0104-1169.0256.2619

2. Ribeiro RM, Pompeo DA, Pinto MH, Ribeiro RC. Coping strategies of nurses in hospital emergency care services. Acta Paul Enferm. 2015; 28(3):21623.doi:http://dx.doi.org/10.1590/19820194201500037

3. Ribeiro MNS, Diniz CX, Perdomo SB, Ribeiro JHS, Barbosa OG, Barros KMSC, et al. Self-esteem and resilience in people with type 2 diabetes mellitus. Mundo Saúde. 2017; 41(2):223-31. doi: http://dx.doi.org/10.15343/0104-7809.20174102223231

4. Sclafani A, Currier P, Chang Y, Eromo E, Raemer D, Miloslavsky EM. Internal medicine residents' exposure to and confidence in managing hospital acute clinical events. J Hosp Med. 2019; 14(4):21823. doi: http://dx.doi.org/10.12788/jhm.3168

5. Barreto DG, Silva KGNS, Moreira SSCR, Silva TS, Magro MCS. Realistic simulation as a teaching strategy: an integrative review. Rev Baiana Enferm [Internet]. 2014 [cited Jun 20, 2019]; 28(2):208-14. Available from: https://portalseer.ufba.br/index. php/enfermagem/article/viewFile/8476/8874

6. Silveira MS, Cogo ALP. The contributions of digital technologies in the teaching of nursing skills: an integrative review. Rev Gaúcha Enferm. 2017; 38(2):e66204. doi: http://dx.doi. org/10.1590/1983-1447.2017.02.66204

7. Van Dyk J, Siedlecki SL, Fitzpatrick JJ. Frontline nurse managers' confidence and self-efficacy. J Nurs Manag. 2016; 24(4):533-9. doi: http://dx. doi.org/10.1111/jonm.12355

8. Pisciottani F, Rocha DF, Costa MR, Figueiredo AE, Magalhães CR. In situ simulation in cardiopulmonary ressuscitation: implications for permanent nursing education. Rev Enferm UFPE on line. 2017; 
11(7):2810-5. doi: http://dx.doi.org/10.5205/reuol.10939-97553-1-RV.1107201722

9. Kurup V, Matei V, Ray J. Role of in-situ simulation for training in healthcare: opportunities and challenges. Curr Opin Anaesthesiol. 2017; 30(6):755-60. doi: http://dx.doi.org/10.1097/ AC 0.000000000000

10. Boling B, Hardin-Pierce M. The effect of high-fidelity simulation on knowledge and confidence in critical care training: an integrative review. Nurse Educ Pract. 2016; 16(1):287-93. doi: http://dx. doi.org/10.1016/j.nepr.2015.10.004

11. Rashid P, Gianduzzo T. Urology technical and nontechnical skills development: the emerging role of simulation. BJU Int. 2016; 117(Suppl 4):9-16. doi: http://dx.doi.org/10.1111/bju.13259

12. Barbeito A, Bonifacio A, Holtschneider M, Segall $\mathrm{N}$, Schroeder R, Mark J et al. In situ simulated cardiac arrest exercises to detect system vulnerabilities. Simul Healthc. 2015;10(3):154-62. doi: dx.doi.org/10.1097/SIH.0000000000000087

13. Andrade PON, Oliveira SC, Morais SCRV, Guedes TG, Melo GP, Linhares FMP. Validation of a clinical simulation setting in the management of postpartum haemorrhage. Rev Bras Enferm. 2019; 72(3):624-631. doi: http://dx.doi. org/10.1590/0034-7167-2018-0065

14. Martins JC, Baptista RC, Coutinho VR, Mazzo A, Rodrigues MA, Mendes IA. Self-confidence for emergency intervention: adaptation and cultural validation of the Self-confidence Scale in nursing students. Rev Latino-Am Enfermagem. 2014; 22(4):554-61. doi: http://dx.doi. org/10.1590/0104-1169.3128.2451

15. Hicks F, Coke L, Li S. Report of findings from the effect of high-fidelity simulation on Nursing students' knowledge and performance: a pilot study. Res Brief [Internet]. 2009 [cited July 20, 2019]; 40. Available from: https://www.worldcat. $\mathrm{org} /$ title/report-of-findings-from-the-effect-ofhigh-fidelity-simulation-on-nursing-studentsknowledge-and-performance-a-pilot-study/ oclc/654567556

16. Fletcher RH, Fletcher SW, Fletcher GS. Epidemiologia clínica - elementos essenciais. Porto Alegre: Artmed; 2014.

17. American Heart Association. Destaques das atualizações específicas de 2017 da American Heart Association para suporte básico de vida em pediatria e para adultos e qualidade de ressuscitação cardiopulmonar [Internet]. 2017 [cited Jun 20, 2019]. Available from: https://eccguidelines.heart. org/wp-content/uploads/2017/12/2017-Focused-Updates_Highlights_PTBR.pdf

18. Zimmermann K, Holzinger IB, Ganassi L, Esslinger $\mathrm{P}$, Pilgrim S, Allen $\mathrm{M}$, et al. Inter-professional in situ simulated team and resuscitation training for patient safety: Description and impact of a programmatic approach. BMC Med Educ. 2015; 15:189. doi: http://dx.doi.org/10.1186/s12909015-0472-5

19. Muniandy RK, Nyein KK, Felly M. Improving the self-confidence level of medical under graduates during emergencies using high fidelity simulation. Med J Malaysia [Internet]. 2015 [cited Jun 20, 2019]; 70(5):300-2. Available from: http:// www.e-mjm.org/2015/v70n5/high-fidelitysimulation.pdf

20. Woods C, West C, Mills J, Park T, Southern J, Usher K. Under graduate student nurses' self-reported prepared ness for practice. Collegian. 2015; 22(4):359-68. foi: http://dx.doi.org/10.1016/j. colegn.2014.05.003 